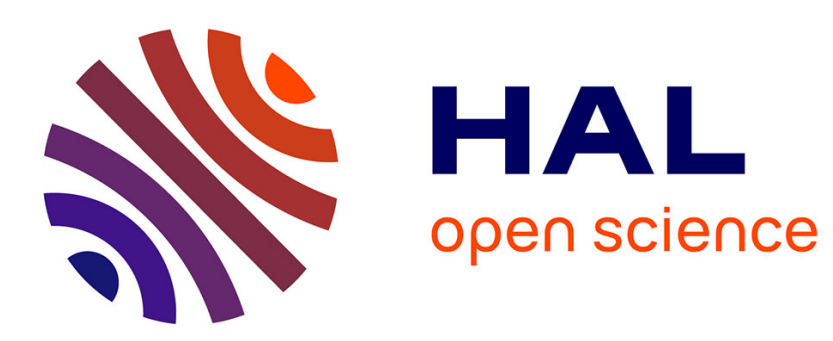

\title{
Derivation of a Langmuir type of model to describe the intrinsic growth rate of gas hydrates during crystallisation from gas mixtures
}

\author{
Jean-Michel Herri, Matthias Kwaterski
}

\section{- To cite this version:}

Jean-Michel Herri, Matthias Kwaterski. Derivation of a Langmuir type of model to describe the intrinsic growth rate of gas hydrates during crystallisation from gas mixtures. Chemical Engineering Science, 2012, 81, pp.28-37. 10.1016/j.ces.2012.06.016 . emse-00724388

HAL Id: emse-00724388

https://hal-emse.ccsd.cnrs.fr/emse-00724388

Submitted on 20 Aug 2012

HAL is a multi-disciplinary open access archive for the deposit and dissemination of scientific research documents, whether they are published or not. The documents may come from teaching and research institutions in France or abroad, or from public or private research centers.
L'archive ouverte pluridisciplinaire HAL, est destinée au dépôt et à la diffusion de documents scientifiques de niveau recherche, publiés ou non, émanant des établissements d'enseignement et de recherche français ou étrangers, des laboratoires publics ou privés. 


\title{
Derivation of a Langmuir type of model to describe the intrinsic growth rate of gas hydrates during crystallization from gas mixtures
}

\author{
Jean-Michel Herri ${ }^{*}$ and Matthias Kwaterski
}

Ecole Nationale Supérieure des Mines de Saint-Etienne, 158 Cours Fauriel, 42023 SaintEtienne, France

\section{Abstract}

Gas Hydrates are crystalline water based solids composed of a three dimensional network of water molecules. They form a network of cavities in which molecules of light gases can be encapsulated depending on their size and affinity. Gas hydrates can by their nature not be classified as chemical compounds since they do not possess a definite stoechiometry. In contrast they have to be regarded as solid solution phases, the stoechiometry of which is not fixed but depends on the composition of the surrounding liquid. At equilibrium, the composition dependence of the hydrate phase can be described by means of the classical van der Waals and Platteeuw model [1]. In the framework of the model, Langmuir constants are used for expressing the relative ability of light components to get enclathrated within the cavities. The work consists in considering again the enclathration of host species, not at thermodynamic equilibrium, but during the crystallization process taking place under nonequilibrium conditions. It aims at proposing a new formulation for the hydrate composition as a function of new intrinsic constants which are based on Langmuir kinetic constants.

\footnotetext{
Corresponding author. Tel: +33 (0)4 774202 92; fax: +33 (0)4 774996 94. E-mail adress: herri@emse.fr (J.M. Herri).
} 


\section{Keywords}

Gas Hydrates, Crystallisation, Thermodynamics, Kinetics, Modelling

\section{Introduction}

Clathrates are solid phases in which a solid network of hydrogen-bonded molecules is organised to generate an internal structure of nanometric cavities capable of encapsulating other components. If the solid network is a water-based network, and if the encapsulated molecules are gas molecules, the corresponding phases are referred to as "gas hydrates". From the sixties, the investigation of gas hydrates, and in particular the thermodynamics of gas hydrates, has been the subject of intense research activities, with respect to both experimental studies as well as modelling in order to understand and prevent the formation of hydrates in oil pipelines. In fact, the combination of light hydrocarbons and water, from the gas phase, or from the liquid phase, can be observed both in gas production and oil production processes.

The thermodynamics of hydrate formation/dissociation has been well understood and a model description has been established by the fundamental work of van der Waals and Platteeuw [1]. The originality of their model [1] is to consider light molecules as adsorbed molecules, and to consider the hypothetical empty network of water molecules providing the cavities as a solid acting as the adsorbent. The light molecules are distributed in the cavities with respect to their relative affinity. The relative affinity between a guest atom or molecule and the water molecules forming the cavity is quantified by the respective Langmuir constant. The Langmuir constant has been tabulated from a physical description which depends on the interaction potential between the guest molecule and the structure. The overall structure is physically stable once the cavities are filled to a sufficiently great extend.

The fact that the empty cavities of the lattice structure are capable of encapsulating light molecules can be used as a profitable property to separate molecules, for example from gas 
mixtures. The original idea has been developed in order to separate molecules that could not be separated by classical distillation. From the beginning of the 1990s, the concept has moved towards the capture of $\mathrm{CO}_{2}$. Many laboratories have recently focused on the investigation of the $\mathrm{CO}_{2} / \mathrm{N}_{2}$ and the $\mathrm{CO}_{2} / \mathrm{CH}_{4}$ gas mixture, respectively, with respect to both, post combustion separation applications and natural gas production.

The present paper has been motivated to explore theoretical considerations which follow from the fact that the hydrate crystallisation is a non-equilibrium process. Whereas the total quantity of gas to be encapsulated in order to stabilise the structure physically is fixed by thermodynamic considerations, the relative composition of the hydrate is governed by the kinetics of the crystallisation process. The hydrate composition becomes dependent on the classical Langmuir constant, but also on new intrinsic constants. Finally, the hydrate composition during crystallization becomes dependent on the geometry of the reactor in which the experiment is being performed. In fact, the hydrate composition depends on the composition of the bulk phase in a way which is in detail outlined in this work. The bulk composition in turn depends on the coupling between the gas consumption rate at the surface of the crystal and the mass transfer rates at the boundary of the bulk phase. The overall modelling of the process is based on the (simultaneous) modelling of all those single elementary steps. It would require taking into account population balances in order to model nucleation, growth, agglomeration, and mass transfer to describe the gas absorption at the gas/liquid interface. This paper focuses on the modelling of the growth rate only and does not deal with this global modelling which has been reviewed in detail by Ribeiro and Lage [2].

In conclusion, the paper presents a new description of the growth rate and hydrate composition during crystallisation. The first part of the document is devoted to the description of the structure of the hydrate and to the definition of the intrinsic rate of integration of the gaseous species into different types of cavities, based on intrinsic kinetic constants describing the Langmuir type of adsorption. The second part deals with the classical description of the 
thermodynamics of the hydrate formation. However, the resulting expressions are modified by consideration of the non-equilibrium nature of the hydrate crystal growth. Finally, a mathematical approach is proposed to solve the coupled equations accounting for mass transfer (diffusion around crystals) and intrinsic growth rates.

\section{Hydrate structure}

Clathrates are ice-like solid phases in which the water molecules form a three-dimensional network. The underlying crystallographic organisation is based on H-bonds between the constituting water molecules. The clathrates of water are also designated improperly as "porous ice" because the water molecules form a solid network of cavities in which the molecules of gases, volatile liquids or other small molecules can be captured. Each structure is a combination of different types of polyhedra sharing faces between them. Jeffrey [3] suggested the nomenclature $e^{f}$ to describe each of these polyhedra: $e$ is the number of edges of the face, and $f$ is the number of faces with $e$ edges. Currently, three different structures, called sI, sII and sH, have been precisely determined experimentally. A complete description of the structures, the characteristics of which are compiled in Table 1, can be found in the books of Sloan and Sloan and Koh [4,5]. For example, structure sI is a cubic structure, the unit cell of which is composed of 42 water molecules, where the cavities possess a radius of $1.2 \mathrm{~nm}$. It provides a network composed of two pseudo spherical cavities $5^{12}$ and six cavities $5^{12} 6^{2}$ which is capable of encapsulating gas molecules of a size and dimension being compatible with the respective internal radius of the cavities of $0.395 \mathrm{~nm}$ and $0.433 \mathrm{~nm}$. 


\begin{tabular}{|c|c|c|c|c|c|c|c|}
\hline & \multicolumn{2}{|c|}{ sI } & \multicolumn{2}{|c|}{ sII } & \multicolumn{3}{|c|}{ sH } \\
\hline Cavity & $5^{12}$ & $5^{12} 6^{2}$ & $5^{12}$ & $5^{12} 6^{4}$ & $5^{12}$ & $4^{3} 5^{6} 6^{3}$ & $5^{12} 6^{8}$ \\
\hline Number of cavities & 2 & 6 & 16 & 8 & 3 & 2 & 1 \\
\hline Average cavity radius $(\mathrm{nm})^{\mathrm{a}}$ & 0.395 & 0.433 & 0.391 & 0.473 & 0.391 & 0.406 & 0.571 \\
\hline Variation in radius, $\%^{\mathrm{b}}$ & 3.4 & 14.4 & 5.5 & 1.73 & & & \\
\hline Coordination number & 20 & 24 & 20 & 28 & 20 & 20 & 36 \\
\hline Number of water molecules & \multicolumn{2}{|c|}{42} & \multicolumn{2}{|c|}{136} & \multicolumn{3}{|c|}{134} \\
\hline Cell parameter a (nm) & \multicolumn{2}{|c|}{$1.1956^{\mathrm{c}}$} & \multicolumn{2}{|c|}{$1.7315^{\mathrm{d}}$} & \multicolumn{3}{|c|}{1.2217} \\
\hline Cell parameter b (nm) & & & & & \multicolumn{3}{|c|}{$1.0053^{\mathrm{e}}$} \\
\hline Cell volume $\left(\mathrm{nm}^{3}\right)$ & \multicolumn{2}{|c|}{$1.709^{c}$} & \multicolumn{2}{|c|}{$5.192^{\mathrm{d}}$} & \multicolumn{3}{|c|}{$1.22994^{\mathrm{e}}$} \\
\hline
\end{tabular}

\footnotetext{
${ }^{\mathrm{a}}[4]$, p. 33.

${ }^{\mathrm{b}}$ Variation in distance of oxygen atoms from centre of cages ([4], p. 33).

${ }^{\mathrm{c}}$ For ethane hydrate [6].

${ }^{\mathrm{d}}$ For tetrahydrofuran hydrate, from [6].

${ }^{\mathrm{e}}$ For methylcyclohexane-methane hydrate [6].
}

\section{Langmuir approach describing the enclathration during crystallization derived from kinetic considerations}

The classical approach of van der Waals and Platteeuw [1] provides a description of the thermodynamics of equilibrium involving clathrate hydrate phases. In this approach, the encapsulation of gas molecules in the empty cavities is described similarly to the adsorption 
of molecules on a two dimensional surface. The model assumptions lead to a Langmuir type of equation for describing this "adsorption" of guest species onto the empty lattice sites.

Different cases may be distinguished, depending on the degree of complexity of the system with regard to the presence of different types of guest species and different types of cavities in the empty hydrate lattice. The simplest case considered deals with a hydrate system containing a single type of guest species $j=j_{0}$ and a single type of cavity $i=i_{0}$. In the second case, hydrate formation in a system containing $N_{\mathrm{g}}$ different potential guest species $j=1, \ldots, N_{\mathrm{g}}$ in a single type of cavity $i=i_{0}$ is taken into account. The third case treated here generalises the second case in that it accounts for the presence of $N_{\text {cav }}$ different types of cavities $i=1, \ldots, N_{\text {cav }}$ in the empty, metastable hydrate lattice. In the following, the set of indices of the guest components, $\left\{1, \ldots, N_{\mathrm{g}}\right\}$, is designated as $S_{\mathrm{g}}$, whereas the set $\left\{1, \ldots, N_{\text {cav }}\right\}$ counting these indices $i$ is denoted as $S_{\text {cav }}$.

During crystallisation (here in a liquid system), the crystal is assumed to be surrounded by two successive layers: an integration layer and a diffusion layer. The integration layer is the region of volume in which a transition between the solid state and the liquid state occurs. Following the approach of Svandal et al. [7], the integration layer can be considered as a solidification layer. It is described by means of the scalar phase field and a composition variable. The composition of the liquid phase layers with respect to $j=1, \ldots, N_{\mathrm{g}}$ species is characterised by a vector of generalised concentrations $\vec{\zeta}=\left(\zeta_{1}, \ldots, \zeta_{N_{\mathrm{g}}}\right)^{\mathrm{T}}$. For chemical engineering applications, especially applications concerning crystallisation processes in liquids, liquid phase non-idealities are often neglected and the fugacity is replaced by a concentration variable $\zeta_{j}$. In this section and in the remaining parts of this work, the variable $\zeta$ to characterise the composition with respect to the concentration of the guest species is chosen to be the mole fraction $x$. At the interface between the solid and the integration layer, 
the scalar phase field assumes the value 1 (solid state). Furthermore, local equilibrium is assumed. The local equilibrium composition is characterised by the set of mole fractions $x_{j}$ for all $j \in S_{\mathrm{g}}$. Then, in the direction of the second interface, between the integration layer and the diffusion layer, the scalar field varies from 1 (solid state) to 0 (liquid state), and the composition varies from $x_{j}$ to $x_{j \text {, int }}$. In contrast to Svandal et al. [7], a model description of the profiles of the scalar phase field or the composition variables is not given in this work. We only assume that they exist and simplify the problem by introducing Eq. (13) which is described below.

The modelling of the diffusion layer is easier, since it is a liquid, though continuous, phase throughout. The composition varies from $x_{j, \text { int }}$ at the interface between the integration layer and the diffusion layer to $x_{j \text {, bulk }}$ at the interface between the diffusion layer and the liquid bulk phase. A schematic representation of the different regions surrounding the hydrate phase under formation conditions is given in Figure 1.

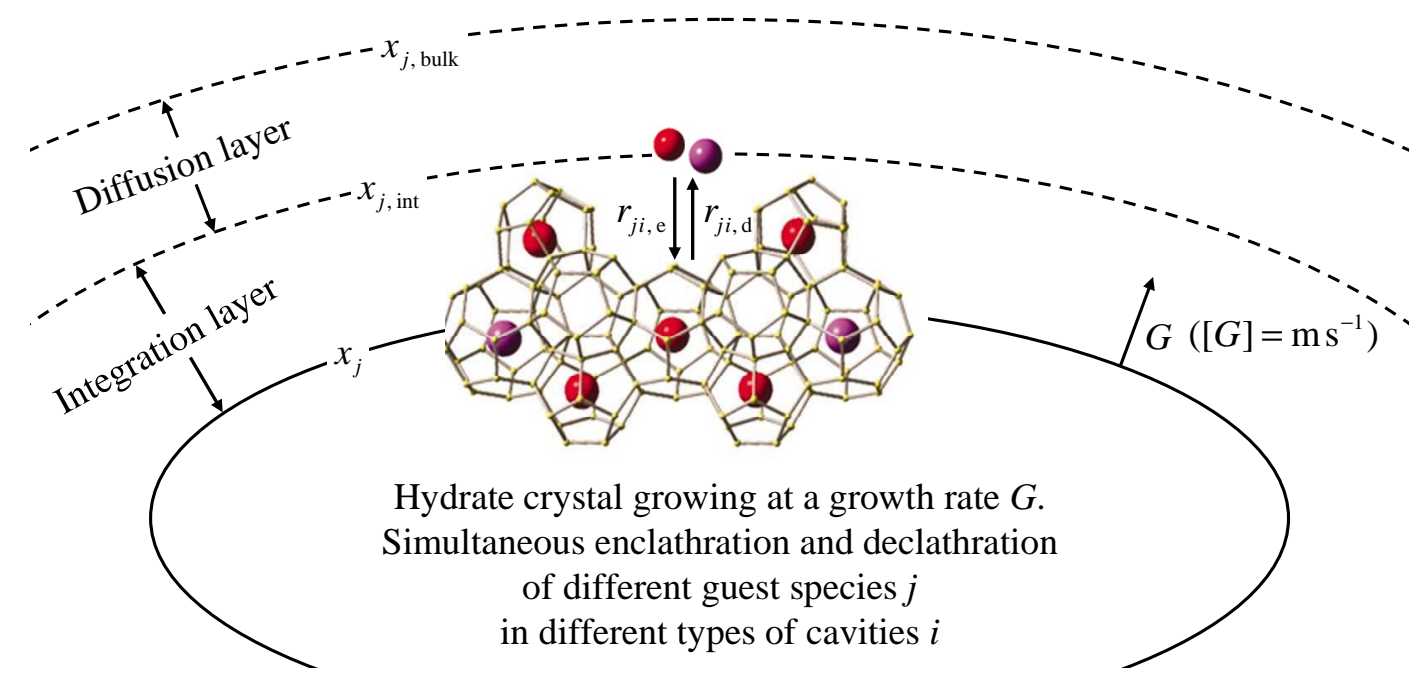

Figure 1. Elementary steps of gas integration in the vicinity of the growing hydrate surface. 
In such a system, the different species $j$ are enclosed by the sites in proportion to their relative affinity. The rate of enclathratation, $r_{j i, \mathrm{e}}$, is directly proportional to the product of the mole fraction $x_{j, \text { int }}$ of $j$ and the fraction of empty cavities, $1-\theta_{i}$, according to

$$
r_{j i, \mathrm{e}}=k_{x, j i, \mathrm{e}} x_{j, \text { int }}\left(1-\theta_{i}\right)
$$

where $k_{x, j i, \mathrm{e}}$ is the corresponding kinetic rate constant of enclathration of species $j$ in a cavity of type $i$. The subscript $x$ at the symbol $k$ refers to the mole fraction as the particular concentration variable chosen for $\zeta$.

The liberation of guest species $j$ from the cavities of type $i$ due to the declathration process can be described by the following rate law

$$
r_{j i, \mathrm{~d}}=k_{j i, \mathrm{~d}} \theta_{j i}
$$

In other words, the corresponding molar rate $r_{j i, \mathrm{~d}}$ is directly proportional to the occupancy factor $\theta_{j i}$, i.e. the fraction of cavities $i$ which are filled with guest species $j$.

As a result, we can define a local flow rate of component $j$ which results from the unbalance between adsorption and desorption:

$$
F_{j i}=r_{j i, \mathrm{e}}-r_{j i, \mathrm{~d}}=k_{x, j i, \mathrm{e}} x_{j, \mathrm{int}}\left(1-\theta_{i}\right)-k_{j i, \mathrm{~d}} \theta_{j i}
$$

Eq. (3) can be rewritten as:

$$
F_{j i}=r_{j i, \mathrm{e}}-r_{j i, \mathrm{~d}}=k_{j i, \mathrm{~d}}\left(\frac{k_{x, j i, \mathrm{e}}}{k_{j i, \mathrm{~d}}} x_{j, \text { int }}(1-\theta)-\theta_{j}\right)
$$

\section{Particular case of equilibrium}

At equilibrium, $F_{j i}=0$, and the following relation is derived from Eq. (4), holding for all $j \in S_{\mathrm{g}}$, taking into account that $x_{j, \text { bulk }} \equiv x_{j, \text { int }} \equiv x_{j}$ : 


$$
k_{j i, \mathrm{~d}}\left(\frac{k_{x, j i, \mathrm{e}}}{k_{j i, \mathrm{~d}}} x_{j}(1-\theta)-\theta_{j}\right)=0,
$$

With the Langmuir constants $C_{x, j i}$ defined as the ratio between the rate of enclathration and declathration according to:

$$
C_{x, j i}=\frac{k_{x, j i, \mathrm{e}}}{k_{j i, \mathrm{~d}}},
$$

Eq. (5), can be re-written as:

$$
\underset{i \in S_{\text {cav }}}{\forall} \underset{j \in S_{\mathrm{g}}}{\forall}\left(C_{x, j i} x_{j}\left(1-\theta_{i}\right)-\theta_{j i}=0\right)
$$

Summing up Eq. (7) over all guest species leads to

$$
\theta_{i}=\frac{\sum_{j^{\prime} \in S_{\mathrm{g}}} C_{x, j^{\prime} i} x_{j^{\prime}}}{1+\sum_{j^{\prime} \in S_{\mathrm{g}}} C_{x, j^{\prime} i} x_{j^{\prime}}} \Leftrightarrow 1-\theta_{i}=\frac{1}{1+\sum_{j^{\prime} \in S_{\mathrm{g}}} C_{x, j^{\prime} i} x_{j^{\prime}}}
$$

Finally, by inserting Eq. (7) into Eq. (8), the following relation is derived for $\theta_{j i}$

$$
\theta_{j i}=\frac{C_{x, j i} x_{j}}{1+\sum_{j^{\prime} \in S_{\mathrm{g}}} C_{x, j^{\prime} i} x_{j^{\prime}}}
$$

\section{Enclathration during crystallisation}

The surface of crystal is supposed to be covered with cavities under formation, which can be regarded as "opened cavities" or active sites. They are assumed to cover the surface and we can define a concentration $\Gamma_{i}$ (number of moles of active cavities of type $i$ per unit of surface area, $\left[\Gamma_{i}\right]=\mathrm{mol} \mathrm{m}^{-2}$ ). Each type of opened cavity $i$ is exposed to a rate $F_{j i}$ (mole of component $j$ /mole of cavity of type $i$ /unit of time). During the growth of the crystals, the 
rate by which the gas molecules $j$ (mole per unit of time) are incorporated into the cavities of the newly created volume is given by:

$$
F_{j i} \Gamma_{i} A_{s}
$$

Where $A_{\mathrm{s}}$ denotes the total surface area of the growing crystals. The crystal is assumed to grow at a rate $G\left([G]=\mathrm{m} \mathrm{s}^{-1}\right)$. The increase in volume of the quantity of solid newly formed per element of time $d t$ is given by

$$
\frac{d V}{d t}=G A_{\mathrm{s}}
$$

The volume of the newly formed solid is composed of water molecules which build a network of cavities of different types $i$. Their molar volumetric concentration is $c_{i}$ (mole of cavity of type $i$ per unit of volume) and they are occupied by gas molecules of type $j$. The occupancy is given by $\theta_{j i}$ (mole of component $j /$ mole of cavity $i$ ). The flow rate by which the gas molecules $j$ (mole per unit of time) are incorporated in the cavities of the new volume is:

$$
c_{i} \theta_{j i} \frac{d V}{d t}=c_{i} \theta_{j i} G A_{\mathrm{s}}
$$

From Eq. (10) and Eq. (12), it follows for all $i \in S_{\text {cav }}$ and all $j \in S_{\mathrm{g}}$ that

$$
\Gamma_{i} F_{j i}=c_{i} \theta_{j i} G
$$

Summing up Eq.(13) over all $j \in S_{\mathrm{g}}$ leads to

$$
\Gamma_{i} F_{i}=c_{i} \theta_{i} G
$$

A general consideration to be taken into account is the following relationship that for each cavity $i$, but for two different molecular adsorbents $j$ and $j^{\prime}$, is obtained from Eq. (13): 


$$
\underset{j \in S_{\mathrm{g}}}{\forall} \underset{j^{\prime} \in S_{\mathrm{g}}}{\forall} \underset{i \in S_{\mathrm{cav}}}{\forall}\left(\frac{F_{j i}}{F_{j^{\prime} i}}=\frac{\theta_{j i}}{\theta_{j^{\prime} i}} \Leftrightarrow \frac{F_{j^{\prime} i}}{\theta_{j^{\prime} i}}=\frac{F_{j i}}{\theta_{j i}}\right)
$$

From Eq. (15) it follows that a characteristic constant $a_{i}$ can be defined according to:

$$
\underset{j \in S_{\mathrm{g}}}{\forall} \underset{i \in S_{\mathrm{cav}}}{\forall}\left(a_{i}=\frac{F_{j i}}{\theta_{j i}}\right)
$$

After summation over all guest species $j$, the total flow rate $F_{i}$ of guest molecules arriving at the cavity $i$ under construction is

$$
F_{i}=\sum_{j \in S_{\mathrm{g}}} F_{j i}=\sum_{j \in S_{\mathrm{g}}} a_{i} \theta_{j i}=a_{i} \sum_{j \in S_{\mathrm{g}}} \theta_{j i}=a_{i} \theta_{i} \quad \Leftrightarrow \quad a_{i}=\frac{F_{i}}{\theta_{i}}
$$

Thus, with Eq. (14) it can be found from Eq. (17) that for all $i \in S_{\text {cav }}$

$$
a_{i}=\frac{c_{i}}{\Gamma_{i}} G
$$

During crystallisation, the cavities of the crystals under formation can encapsulate components in consideration of the unbalance between the declathration and enclathration rate. Therefore, from Eq. (4) and (6), the overall rate $F_{j i}$ by which molecules of type $j$ are accumulated in the cavities of type $i$ is given by

$$
F_{j i}=r_{j i, \mathrm{e}}-r_{j i, \mathrm{~d}}=k_{j i, \mathrm{~d}}\left(C_{x, j i} x_{j, \text { int }}\left(1-\theta_{i}\right)-\theta_{j i}\right) \geq 0
$$

However, once it has been formed and because it has been formed, the hydrate can be considered being in equilibrium with the integration layer at composition $x_{j}$. For this phase, all of the conditions characterising a state of equilibrium and especially:

$$
\theta_{j i}=C_{x, j i} x_{j}\left(1-\theta_{i}\right)
$$


are assumed to hold. Therefore, upon substituting the right hand side of Eq. (20) for $\theta_{j i}$ in Eq. (19) one arrives at

$$
F_{j i}=r_{j i, \mathrm{e}}-r_{j i, \mathrm{~d}}=k_{j i, \mathrm{~d}} C_{x, j i}\left(x_{j, \text { int }}-x_{j}\right)\left(1-\theta_{i}\right)
$$

Another way to describe the problem is to start again from Eq. (19) and to write it in the form

$$
\frac{F_{j i}}{k_{j i, \mathrm{~d}}}=C_{x, j i} x_{j, \mathrm{int}}\left(1-\theta_{i}\right)-\theta_{j i}
$$

Combining Eq. (22) with

$$
\theta_{j i}=\frac{F_{j i}}{a_{i}}
$$

leads to the following identity

$$
\left(\frac{1}{k_{j i, \mathrm{~d}}}+\frac{1}{a_{i}}\right) F_{j i}=C_{x, j i} x_{j, \mathrm{int}}\left(1-\theta_{i}\right) \Leftrightarrow F_{j i}=\frac{C_{x, j i} x_{j, \mathrm{int}}\left(1-\theta_{i}\right)}{1 / k_{j i, \mathrm{~d}}+1 / a_{i}},
$$

being valid for all $j \in S_{\mathrm{g}}$ and $i \in S_{\text {cav }}$. The equality of Eq. (21) and Eq. (24) reads

$$
\frac{C_{x, j i} x_{j, \text { int }}}{1 / k_{j i, \mathrm{~d}}+1 / a_{i}}=k_{j i, \mathrm{~d}} C_{x, j i}\left(x_{j, \text { int }}-x_{j}\right),
$$

which is equivalent to

$$
x_{j, \text { int }}-x_{j}=\frac{x_{j, \text { int }}}{1+k_{j i, \mathrm{~d}} / a_{i}}
$$

Eq. (26) has a strong consequence. The left member of the equality in Eq.(26) is a driving force, expressed here by a difference of the mole fraction of the species to be integrated in the structure under growing. This driving force is the same for any cavities. It means that the right member of the equality in Eq.(26) is also independent of the cavity. It can be stated more 
specifically that the ratio $k_{j i, \mathrm{~d}} / a_{i}$ is independent of the nature of the cavity. Upon expressing $a_{i}$ by means of Eq. (18) in terms of $c_{i}, \Gamma_{i}$ and $G$, the ratio $k_{j i, \mathrm{~d}} / a_{i}$ is given by

$$
\frac{k_{j i, \mathrm{~d}}}{a_{i}}=\frac{k_{j i, \mathrm{~d}} \Gamma_{i}}{G c_{i}}
$$

The independence of $k_{j i, \mathrm{~d}} / a_{i}$ of the nature of the cavity $i$ gives rise to the definition of a kinetic constant $k_{j}$, which is to be regarded as an intrinsic kinetic constant of component $j$

$$
k_{j}=k_{j i, \mathrm{~d}} \frac{\Gamma_{i}}{c_{i}}
$$

By expressing $k_{j i, \mathrm{~d}} / a_{i}$ in Eq. (26) by means of Eq. (27) and (28) in terms of $k_{j}$ and $G$ and solving for $x_{j}$, the following relationship is obtained

$$
x_{j}=x_{j, \text { int }} \frac{k_{j} / G}{1+k_{j} / G}
$$


Table 2 summarises the relations that allow for the calculation of the hydrate composition as a function of the composition of the liquid phase in the vicinity of the growing hydrate crystal. The expressions are dependent on the composition $x_{j \text {,int }}$ at the interface between the integration layer and the diffusion layer.

To composition can be evaluated from a mass balance in Eq. (30) giving the equality between the integration rate due to the Langmuir type of enclathration (lef-hand-side), and the gas diffusion around (right-hand-side):

$$
\underset{j \in S_{\mathrm{g}}}{\forall}\left(G A_{\mathrm{s}} \sum_{i \in S_{\mathrm{cav}}} c_{i} \theta_{j i}=d_{j}^{*} A_{\mathrm{s}}\left(x_{j, \mathrm{bulk}}-x_{j, \mathrm{int}}\right) \frac{\rho_{\mathrm{w}}^{\circ}}{M_{\mathrm{w}}}\right),
$$

where $x_{j, \text { bulk }}$ is the mole fraction of $j$ in the bulk phase, and $d_{j}^{*}\left(\left[d_{j}^{*}\right]=\mathrm{ms}^{-1}\right)$ the mass transfer coefficient of the guest species $j$ around the crystal, respectively. $\rho_{\mathrm{w}}^{\circ}$ and $M_{\mathrm{w}}$, respectively, stands for the density $\left(\left[\rho_{\mathrm{w}}^{\circ}\right]=\mathrm{kg} \mathrm{m}^{-3}\right)$ and the molar mass $\left(\left[M_{\mathrm{w}}\right]=\mathrm{g} \mathrm{mol}^{-1}\right)$ of the solvent (water), respectively. $d_{i}^{*}$ can be estimated from a classical correlation between the dimensionless Reynolds, Sherwood and Schmidt numbers of/around the crystal particle (index "p"), $\mathrm{Re}_{\mathrm{p}}, \mathrm{Sh}_{\mathrm{p}}$ and $\mathrm{Sc}$, as for example by the one of Armenante and Kirwan [8] which can be retained

$$
\begin{gathered}
\mathrm{Sh}_{\mathrm{p}}=2+0.52 \mathrm{Re}_{\mathrm{p}}^{0.52} \mathrm{Sc}^{1 / 3} \\
\mathrm{Sh}_{\mathrm{p}}=\frac{d_{j}^{*} l}{D_{j}}, \operatorname{Re}_{\mathrm{p}}=\frac{l^{4 / 3} \varepsilon^{1 / 3}}{v}, \mathrm{Sc}=\frac{v}{D_{j}}
\end{gathered}
$$

In the equations compiled in Eq. (32), $l$ is the diameter of the crystals under growing and $v$ the kinematic viscosity of the liquid phase, approximated by the kinematic viscosity of the pure solvent, i.e. water. $D_{j}\left(\left[D_{j}\right]=\mathrm{m}^{2} \mathrm{~s}^{-1}\right)$ denotes the diffusivity of the gas in the solvent. It can be extrapolated from the value at a given temperature by using the correlation of Wilke 
and Chang (1995) [9] in which $D_{j} \eta / T=$ const . $\eta$ is the dynamic viscosity at temperature $T$, $\varepsilon$ stands for the energy dissipation rate per unit mass of the fluid, here water. For the case of a stirred reactor equipped with a four blades impeller, the following relationship for $\varepsilon$ is provided by Baldi et al. [10].

$$
\varepsilon=\frac{(\text { stirring rate })^{5}(\text { impeller diameter })^{3}}{\text { liquid volume }}
$$


Table 2 Occupancy factor of enclathrated molecules as a function of the composition in the liquid phase. The equations of the right-hand column are obtained from the classical Langmuir expressions from Eq. (9) (left column) upon replacing $x_{j}$ by the expression of Eq. (29) (right column).

\begin{tabular}{|c|c|c|}
\hline & $\begin{array}{l}\text { Thermodynamic } \\
\text { equilibrium }^{\mathrm{a}}\end{array}$ & $\begin{array}{l}\text { Local equilibrium resulting from a kinetic } \\
\qquad \text { equilibrium }^{\mathrm{a}}\end{array}$ \\
\hline$\theta_{j i}$ & $\frac{C_{x, j i} x_{j}}{1+\sum_{j^{\prime} \in S_{\mathrm{g}}} C_{x, j^{\prime} i} x_{j^{\prime}}}$ & $\frac{K_{j} C_{x, j i} x_{j, \text { int }} /\left(1+k_{j} / G\right)}{1+\sum_{j^{\prime} \in S_{\mathrm{g}}} K_{j^{\prime}} C_{x, j^{\prime} i} x_{j^{\prime}, \text { int }} /\left(1+k_{j^{\prime}} / G\right)}$ \\
\hline$\theta_{i}$ & $\frac{\sum_{j \in S_{g}} C_{x, j i} x_{j}}{1+\sum_{j^{\prime} \in S_{\mathrm{g}}} C_{x, j^{\prime} i} x_{j^{\prime}}}$ & $\frac{\sum_{j \in S_{\mathrm{g}}} K_{j} C_{x, j i} x_{j, \text { int }} /\left(1+k_{j} / G\right)}{1+\sum_{j^{\prime} \in S_{\mathrm{g}}} K_{j^{\prime}} C_{x, j^{\prime} i} x_{j^{\prime}, \text { int }} /\left(1+k_{j^{\prime}} / G\right)}$ \\
\hline $1-\theta_{i}$ & $\frac{1}{1+\sum_{j \in S_{\mathrm{g}}} C_{x, j i} x_{j}}$ & $\frac{1}{1+\sum_{j \in S_{\mathrm{g}}} K_{j} C_{x, j i} x_{j, \text { int }} /\left(1+k_{j} / G\right)}$ \\
\hline
\end{tabular}

${ }^{\text {a }}$ The Langmuir coefficient is usually calculated by using a modified Kihara approach in which the mole fraction $x_{j}$ of the guest component $j$ is replaced by the corresponding fugacity $f_{j}$. Expressing the relation between the Langmuir coefficients $C_{x, j i}$ and $C_{f, j i}=C_{j i}$ as $C_{x, j i} x_{j}=C_{j i} f_{i}$, an approximate relation can be derived for calculating $C_{x, j i}$ as a function of $C_{j i}$ by using a simplified version of Henry's law in the form of $f_{j}=x_{j} k_{H, j \mathrm{w}}^{\infty} \exp \left(p V_{\mathrm{m}, j}^{\infty} / R T\right)$, where liquid phase non-idealities expressed by means of the activity coefficient as well as the pressure dependence of $V_{\mathrm{m}, j}^{\infty}$, the partial molar volume of $j$ at infinite dilution, are neglected. In this relationship $k_{H, j \mathrm{w}}^{\infty}$ is Henry's constant of the guest species $j$ in the solvent water at the saturation pressure of the solvent. By proceeding in that way, the approximate relation $C_{x, j i}=C_{j i} k_{H, j \mathrm{w}}^{\infty} \exp \left(p V_{\mathrm{m}, j}^{\infty} / R T\right)$ is obtained. 
Thus, once numerical values for $k_{j}\left(\left[k_{j}\right]=\mathrm{ms}^{-1}\right)$ and for $G$ are assumed, the $x_{j \text {, int }}$ values can be determined as the solution of the system of the $N$ non-linear equations Eq. (34).This set of equations is obtained by substituting the expressions for $\theta_{j i}$ of Table 2 into Eq (30)

$$
\underset{j \in S_{\mathrm{g}}}{\forall}\left(G \sum_{i \in S_{\mathrm{cav}}} c_{i} \frac{K_{j} C_{x, j i} x_{j, \text { int }} /\left(1+K_{j}\right)}{1+\sum_{j^{\prime} \in S_{\mathrm{g}}} K_{j^{\prime}} C_{x, j^{\prime} i} x_{j^{\prime}, \text { int }} /\left(1+K_{j^{\prime}}\right)}=d_{j}\left(x_{j, \text { bulk }}-x_{j, \text { int }}\right)\right) \text { with } d_{j}=d_{j}^{*} \frac{\rho_{\mathrm{w}}^{\circ}}{M_{\mathrm{w}}}
$$

where the quantity $d_{j}$ has the dimension of a molar flux and thus $\left[d_{j}\right]=\mathrm{mol} \mathrm{m}^{-2} \mathrm{~s}^{-1}$. The last equation to be taken into account for completely solving the problem is a relation expressing the hydrate stability. The subject of this work is not to describe the physical model inherited from the model of van der Waals and Platteeuw [1]. For more details on that issue, see $[4,5]$. A fruitful reading could also be our previous study [11] which has motivated the work presented in this article. In fact, it focuses on the inter-dependency of internal parameters (i.e. Kihara parameters versus reference state parameters) and points to the difference between the experimental data from different laboratories.

The fundamental equation expressing the hydrate stability is deduced from statistical thermodynamics. It demonstrates that the hydrates become stable once the cavities are sufficiently filled, without considering the chemical nature of the components.

$$
\frac{\Delta \mu_{\mathrm{w}}^{\beta-\mathrm{H}}(T, p, \vec{\theta})}{R T}=\sum_{i \in S_{\mathrm{cav}}} v_{i} \ln \left(1-\theta_{i}\right)
$$

In Eq. (35), $R$ is the universal molar gas constant and $\vec{\theta}$ is the vector of independent occupancy factors. The summation is to be performed over all types of cavities (e.g., the two types of cavities, $5^{12}$ and $5^{12} 6^{2}$ in case of a sI hydrate with a stoichiometry of 2 and 6 , respectively, as shown in Table 1), counted by the index $i . \Delta \mu_{\mathrm{w}}^{\beta-\mathrm{H}}$ is the difference of the chemical potential of water in the hydrate phase and the chemical potential of water in an 
hypothetical empty hydrate lattice, denoted as $\beta$. It can be calculated since, at equilibrium, the chemical potential of water in the solid phase and in the liquid phase are equal. The difference between the chemical potential of water in the liquid phase and in the $\beta$-phase, $\Delta \mu_{\mathrm{w}}^{\beta-\mathrm{L}}(T, p)$, is calculated by means of the following relation originating from classical thermodynamics, explained in detail in [4,5]

$$
\begin{aligned}
\frac{\Delta \mu_{\mathrm{w}}^{\beta-\mathrm{L}}(T, p, \vec{x})}{R T}= & \frac{\Delta \mu_{\mathrm{w}}^{\beta-\mathrm{L}}\left(T_{0}, p_{0}\right)}{R T_{0}}-\int_{T_{0}}^{T} \frac{\Delta H_{\mathrm{m}, \mathrm{w}}^{\beta-\mathrm{L}}\left(T, p_{0}\right)}{R T^{2}} d T+\int_{p_{0}}^{p} \frac{\Delta V_{\mathrm{m}, \mathrm{w}}^{\beta-\mathrm{L}}(T, p)}{R T} d p \\
& -\ln a_{\mathrm{w}}^{\mathrm{L}}(T, p, \vec{x})
\end{aligned}
$$

In Eq. (36), $T_{0}$ and $p_{0}$ are reference values for temperature and pressure, taken to be 273.15 K and 0 MPa [11], respectively, whereas $\vec{x}$ denotes the vector of independent mole fractions in the liquid phase indicating the composition dependence of $\mu_{\mathrm{w}}^{\beta-\mathrm{L}}$ and $a_{\mathrm{w}}^{\mathrm{L}}$, the latter of which stands for the activity of water in the liquid phase. $\Delta V_{\mathrm{m}, \mathrm{w}}^{\beta-\mathrm{L}}(T, p)$ denotes the difference in the molar volumes of water in the liquid and the $\beta$-phase, respectively. The latter has been measured with high accuracy by von Stackelberg and Müller [12]. The value of $\Delta H_{\mathrm{m}, \mathrm{w}}^{\beta-\mathrm{L}}\left(T, p_{0}\right)$ can be expressed as temperature dependent according to Sloan and Sloan and Koh [4,5]. The last parameter of the equation is $\Delta \mu_{\mathrm{w}}^{\beta-\mathrm{L}}\left(T_{0}, p_{0}\right)$. Numerical values published for this parameter show strong variations among different laboratories [4,5]. Therefore, it needs to be selected with precaution when being used in calculations of the Langmuir coefficient together with Kihara parameters which are retained on the other hand [11]. Once $\mu_{\mathrm{w}}^{\beta-\mathrm{L}}$ is calculated, which in equilibrium is equal to $\Delta \mu_{\mathrm{w}}^{\beta-\mathrm{H}}$, the hydrate composition needs to satisfy the identity given by Eq. (35). Hence, by eliminating $\theta_{i}$ from Eq. (35) via the expression presented in Table 2, the growth rate $G$ fulfils the equation

$$
\frac{\Delta \mu_{\mathrm{w}}^{\beta-\mathrm{H}}}{R T}=-\sum_{i \in S_{\mathrm{cav}}} v_{i} \ln \left(1+\sum_{j \in S_{\mathrm{g}}} \frac{K_{j} C_{x, j i} x_{j, \text { int }}}{1+K_{j}}\right)
$$


Finally, the local equilibrium is defined when the values of $G$ and $x_{j, \text { int }}$ for all $j \in S_{g}$ satisfy Eq. (34) and (37).

The calculation procedure is outlined in more detail in Figure 2. It is a double convergence loop. In the first loop, an iteration is performed on the growth rate in order to satisfy Eq. (37) describing the hydrate stability. From a physical point of view, the $G$ value is the value at which the structure can grow by incorporation of solute gas to such an amount that is sufficient for stabilising the structure (i.e. the cavities are filled to a sufficient extend). The relative proportion to which the different gas molecules $j$ are entering the structure is determined in the second convergence loop which is an indirect consequence of the competition between the diffusion rates around the crystals and integration rates in the structure. By this competition the $x_{j, \text { int }}$ values are fixed.

Even for a system containing only a single hydrate forming component $j \equiv \mathrm{A}$, it can be demonstrated that the hydrate phase does not form at equilibrium. Eq. (35) along with the expressions presented in Table 2, can be written at kinetic equilibrium, during crystallisation, and yields to Eq. (37), and after mathematical reformulation to Eq. (38). At thermodynamic equilibrium, Eq. (35) leads to Eq.(38)

$$
\exp \left(-\frac{\Delta \mu_{\mathrm{w}}^{\beta-\mathrm{H}}}{R T}\right)_{\text {cryst }}=\prod_{i \in S_{\mathrm{cav}}}\left(1+\frac{K_{j} C_{x, j i} x_{j, \mathrm{int}}}{1+K_{j}}\right)^{v_{i}}=\prod_{i \in S_{\mathrm{cav}}}\left(1+C_{x, j i} x_{j}\right)^{v_{i}}
$$

where the index "cryst" indicates that the properties are calculated during crystallisation. $x_{j}$ is to be calculated from $x_{j, \text { int }}$ by means of Eq. (29). At equilibrium, the following relation holds

$$
\exp \left(-\frac{\Delta \mu_{\mathrm{w}}^{\beta-\mathrm{H}}}{R T}\right)_{\mathrm{eq}}=\prod_{i \in S_{\mathrm{cav}}}\left(1+C_{x, j i} x_{j, \mathrm{eq}}\right)^{v_{i}}
$$

On the other hand Eq. (36) becomes: 


$$
\left(\Delta \mu_{\mathrm{w}}^{\beta-\mathrm{L}}\right)_{\mathrm{cyst}}-\left(\Delta \mu_{\mathrm{w}}^{\beta-\mathrm{L}}\right)_{\mathrm{eq}}=\Delta V_{\mathrm{m}, \mathrm{w}}^{\beta-\mathrm{L}}(T)\left(p-p_{\mathrm{eq}}(T)\right)-R T \ln \frac{a_{\mathrm{w}}^{\mathrm{L}}(T, p)}{a_{\mathrm{w}}^{\mathrm{L}}\left(T, p_{\mathrm{eq}}(T)\right)}
$$

Eq. (40) shows that in general the difference $\left(\Delta \mu_{\mathrm{w}}^{\beta-\mathrm{L}}\right)_{\text {cyst }}-\left(\Delta \mu_{\mathrm{w}}^{\beta-\mathrm{L}}\right)_{\mathrm{eq}}$ does not vanish. Since the fundamental equation describing the equilibrium condition is $\Delta \mu_{\mathrm{w}}^{\mathrm{L}-\beta}=\Delta \mu_{\mathrm{w}}^{\mathrm{H}-\beta}$, it follows that $\left(\Delta \mu_{\mathrm{w}}^{\beta-\mathrm{H}}\right)_{\mathrm{cyst}}-\left(\Delta \mu_{\mathrm{w}}^{\beta-\mathrm{H}}\right)_{\mathrm{eq}}$ is also different from zero, and hence, it is found that

$$
\begin{gathered}
\prod_{i \in S_{\mathrm{cav}}}\left(1+C_{x, j i} x_{j}\right)^{v_{i}} \neq \prod_{i \in S_{\mathrm{cav}}}\left(1+C_{x, j i} x_{j, \mathrm{eq}}\right)^{v_{i}} \\
x_{j} \neq x_{j, \mathrm{eq}}
\end{gathered}
$$

Even for gas hydrate formed in a system containing a single guest component $j \equiv \mathrm{A}$, the hydrate which crystallizes from the liquid solution is in kinetic equilibrium with a liquid layer the composition of which $\left(x_{j}\right)$ is not identical with the equilibrium composition $x_{j, \text { eq }}$. 


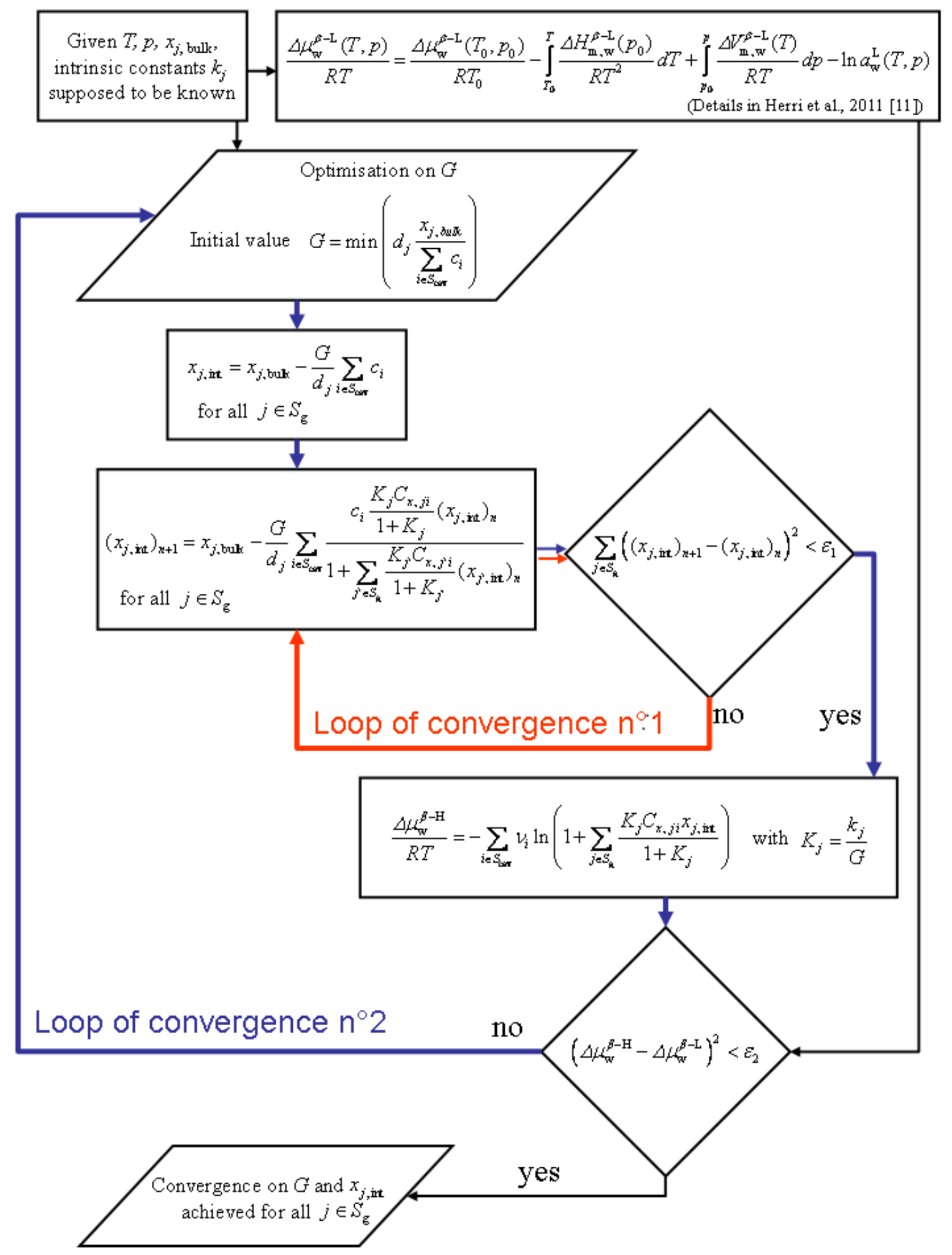

Figure 2 Double procedure of convergence to calculate the gas hydrate growing rate G, and the gas composition $x_{j \text {, int }}$ around the growing hydrate. 


\section{Discussion}

The approach which has been developed here needs to be compared to the pioneering works of Englezos et al. [13,14]. In contrast to our model, Englezos et al have postulated that the integration of any of the hydrate forming guest species is independent from the integration of all the other guest species. He assumed a global intrinsic reaction rate $R$ defined according to:

$$
R=\sum_{j \in S_{\mathrm{g}}} K_{j}^{*}\left(f_{j}-f_{j, \text { eq }}\right)
$$

where

$$
\frac{1}{K_{j}^{*}}=\frac{1}{k_{j}^{*}}+\frac{1}{D_{j}}
$$

with $K_{j}^{*}$ and $D_{j}$ being, respectively, the intrinsic kinetic constant and the diffusion constant of component $j$ around the growing crystals, respectively. The difference $f_{j}-f_{j \text {, eq }}$ is the driving force, conventionally taken as the difference between the fugacity in the vicinity of the growing crystals and the fugacity of the hydrate at equilibrium. One of the important assumptions of the model of Englezos et al. [14] is that the equilibrium fugacities are calculated independently, although the authors do not give a clear instruction of how they are exactly to be calculated.

For a single gas hydrate, this equation has the advantage to simplify to a popular model in crystallization, known under the acronym of BCF model (from Burton, Cabrera and Frank [15]). In this model, the linear dependence of the growth rate to the driving force corresponds to the growth of a rough surface. It can be further assumed that the overall mechanism of the coupled processes of integration and diffusion exhibits a first order dependence on the fugacity. Moreover, for a single gas, there is no competition between the different components to occupy the cavities up to the adequate filling level which stabilizes the structure physically and chemically. 
For gas mixtures, the model of Englezos et al. $[13,14]$ considers that the molar flux of molecules is the sum of independent individual fluxes. However, the model does not describe the local hydrate stability during crystallization as a consequence of the crystallization itself, which instead needs to be assumed independently. Neither the papers of Englezos et al. $[13,14]$ are clear on that point, nor more recent work [16] that has inherited features from the model of Englezos et al. [13,14]. The authors [16] state that the hydrate composition is calculated by using a particular software (MEGHA software) without giving a detailed description of the vector of mole fractions of the guest molecules $\vec{x}=\left(x_{1}, \ldots, x_{N_{\mathrm{g}}}\right)^{\mathrm{T}}$ which is being used in calculating the occupancy factors of the cavities, as for example in Eq. (8) and (9) with $\zeta \equiv x$.

In the approach defended here, the local hydrate stability is coupled with the crystallization itself. The competition between gas molecules, firstly at the diffusion layer scale and secondly at the integration layer scale, is considered as the fundamental rule to describe the growth rate under non-equilibrium conditions. The hydrate stability, i.e., the mole fraction at equilibrium $x_{j}$, becomes intrinsically dependent on the crystallisation mechanisms and kinetic rates. As a result, both the hydrate composition and the growth rate become dependent on the intrinsic kinetic constants (Figure 2).

\section{Numerical application}

We have tested the model against $\mathrm{CO}_{2}-\mathrm{N}_{2}$ hydrate. The underlying gas mixture is an example for a mixture as it is typically encountered in the separation of flue gases emitted in combustion processes. $\mathrm{CO}_{2}$ is highly soluble in comparison to $\mathrm{N}_{2}$. The solubilities are calculated by means of a Henry's law approach as described in the monograph of Sloan (1998), p. 250ff [4], using the parameters of the corresponding correlation equation for $\mathrm{CO}_{2}$ and $\mathrm{N}_{2}$ as given in the same literature source on p. 253, [4]. The respective diffusion constants 
of the two gases in water, $D_{\mathrm{CO}_{2}}$ and $D_{\mathrm{N}_{2}}$, at the temperature of $1{ }^{\circ} \mathrm{C}$ have been calculated from the correlation of Wilke and Chang (1995) [9], using as initial values the corresponding coefficients at ambient temperature, $D_{\mathrm{CO}_{2}}(T=298.15 \mathrm{~K})=2.00 \times 10^{-5} \mathrm{~cm}^{2} \mathrm{~s}^{-1} \quad$ [9] and $D_{\mathrm{N}_{2}}(T=298.15 \mathrm{~K})=1.9 \times 10^{-5} \mathrm{~cm}^{2} \mathrm{~s}^{-1}$ [17]. The crystallization is supposed to be performed in a reactor containing $1 \mathrm{dm}^{3}$ of water which is agitated by means of a four blades vertical stirrer of $0.058 \mathrm{~m}$ diameter. The temperature is assumed to be set to $1^{\circ} \mathrm{C}$. Under such conditions we have observed [18] that methane hydrate particles have a mean diameter in the range of $10 \mu \mathrm{m}$ (at a stirring rate of $400 \mathrm{rpm}$ ) and $24 \mu \mathrm{m}$ (at a stirring rate of $800 \mathrm{rpm}$ ). For the simulation, a stirring rate of $400 \mathrm{rpm}$ and a value of $10 \mu \mathrm{m}$ for the particle diameter have been retained. Using this set of numerical values for the quantities appearing in Eqs. (31)-(34), the values $d_{\mathrm{CO}_{2}}(T=274.15 \mathrm{~K})=47.87 \mathrm{~mol} \mathrm{~m}^{-2} \mathrm{~s}^{-1}$ and $d_{\mathrm{N}_{2}}(T=274.15 \mathrm{~K})=45.73 \mathrm{~mol} \mathrm{~m}^{-2} \mathrm{~s}^{-1}$ are derived. At this stage, the knowledge of these constants allows for performing the numerical calculations with Eq. (34), corresponding to the first loop of convergence in Figure 2. This loop of convergence expresses the mass balance between the species migrating across the diffusion layer around the particles due to diffusive transport and the species being incorporated into the particle.

The second loop of convergence imposes the restriction that the hydrate particle is at local equilibrium with its surrounding integration layer. Therefore a thermodynamic calculation needs to be performed in which the equality of the chemical potential of water in the hydrate and the liquid phase, using the expressions given in Eqs. (36) and (37), is verified. The numerical values of the thermodynamic constants are taken from [11].

Figure 3 concerns the crystallisation of structure I gas hydrates formed from a liquid solution in equilibrium with a gas phase composed of an equimolar mixture of $\mathrm{CO}_{2}-\mathrm{N}_{2}$. In this example, a temperature of $1^{\circ} \mathrm{C}$ is assumed. The equilibrium pressure of the mixture is 2.584 $\mathrm{MPa}$ and the composition of the hydrate at equilibrium is $85.6 \% \mathrm{CO}_{2}$. The equilibrium 
pressure for pure $\mathrm{CO}_{2}$ hydrate is $1.407 \mathrm{MPa}$, while for pure $\mathrm{N}_{2}$ hydrate a value of $17.438 \mathrm{MPa}$ is obtained. That means that a pure $\mathrm{CO}_{2}$ or pure $\mathrm{N}_{2}$ gas hydrate can form from the equimolar gas mixture only if the partial pressure is respectively superior to twice $1.407 \mathrm{MPa}$ or twice 17.438 MPa.

The simulation is performed at a pressure of $4 \mathrm{MPa}$. Figure 3 plots the composition of the hydrate as a function of the kinetic constants $k_{\mathrm{CO}_{2}}$ and $k_{\mathrm{N}_{2}}$ as defined in Eq. (28). In Figure 4 the growth rate $G$ is plotted.

At high values of $k_{\mathrm{N}_{2}}$ and $k_{\mathrm{CO}_{2}}$ the growth rate attains a maximum value of $0.64 \mu \mathrm{m} \mathrm{s}^{-1}$ which remains constant over an extended interval of $k_{\mathrm{N}_{2}}$ and $k_{\mathrm{CO}_{2}}$ and which is limited by the diffusion around the particles. In such a case, we observe that the composition of the hydrate remains constant, close to a value of $85.5 \% \mathrm{CO}_{2}$. This value is extremely closed to the value corresponding to the crystallisation of gas hydrate at equilibrium $\left(1^{\circ} \mathrm{C}, 2.584 \mathrm{MPa}\right)$ in which the composition is $85.6 \% \mathrm{CO}_{2}$.

Decreasing the kinetic rate of integration of nitrogen or carbon dioxide in the hydrate phase ( $k_{\mathrm{N}_{2}}$ or $k_{\mathrm{CO}_{2}}$ ) is equivalent to diminish the consumption of one of the gases. It results in an enrichment of the hydrate in the other gas. At a pressure of $4 \mathrm{MPa}$ and equimolar gas composition, the pure $\mathrm{CO}_{2}$ hydrate is stable. So, the deactivation of the nitrogen integration results in the formation of a pure $\mathrm{CO}_{2}$ hydrate. But, at this pressure, the pure nitrogen hydrate is not stable and the deactivation of $\mathrm{CO}_{2}$ integration can not lead to the formation of a pure $\mathrm{N}_{2}$ hydrate. At a pressure of $4 \mathrm{MPa}$, the hydrate containing the lowest relative amount of $\mathrm{CO}_{2}$ which can be formed contains $80 \%$ of $\mathrm{CO}_{2}$. The deactivation of $\mathrm{CO}_{2}$ needs to be compensated on the $\mathrm{N}_{2}$ side. This is achieved from both a decrease of the diffusion and growth rate, respectively, as a consequence of Eq. (34). 


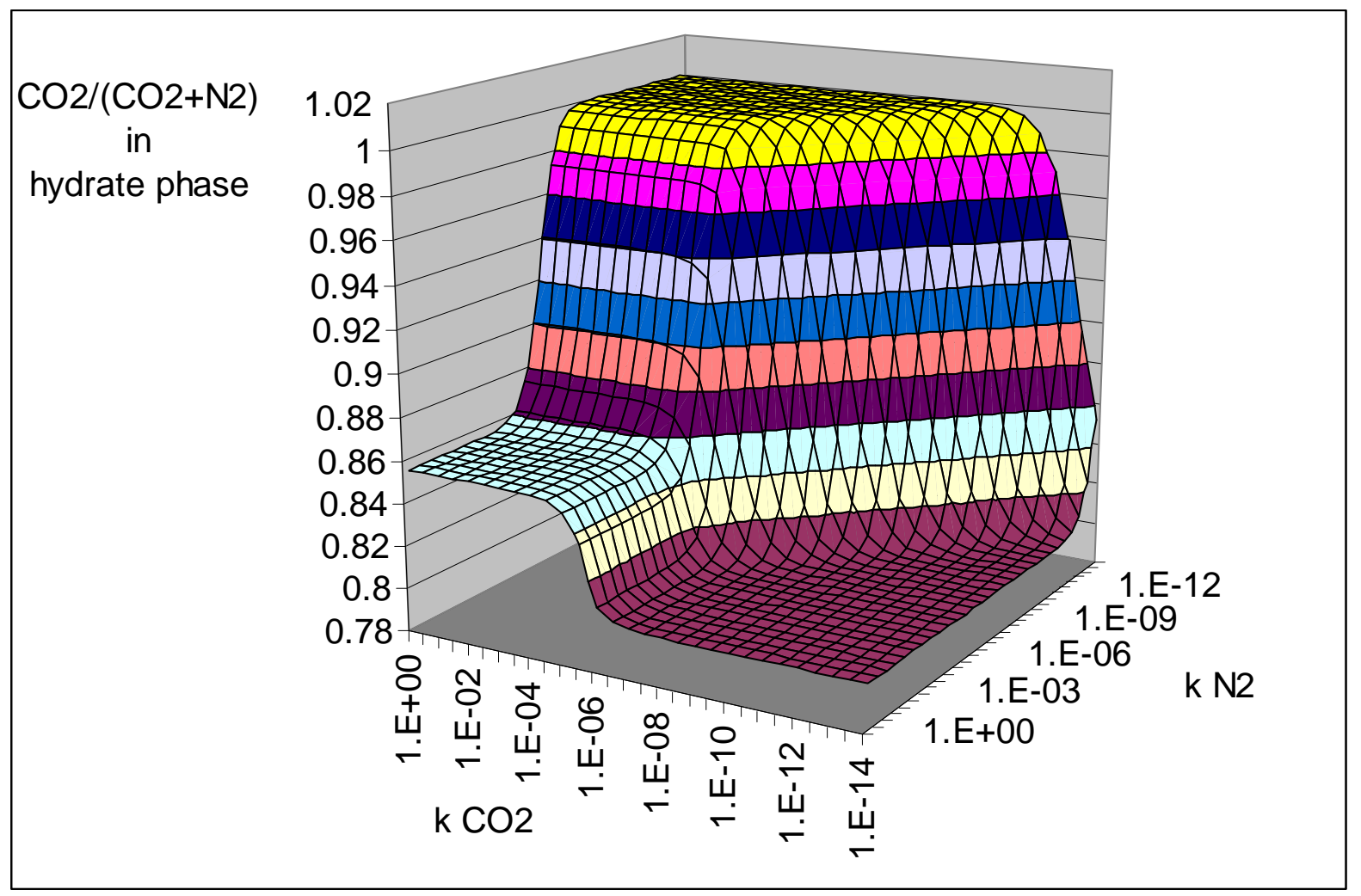

Figure 3 Molar composition of the hydrate phase as a function of the intrinsic growth rates. The liquid phase is supposed to be in equilibrium with a gas phase composed of an equimolar $\mathrm{CO}_{2}-\mathrm{N}_{2}$ mixture, at a pressure of $4 \mathrm{MPa}$ and temperature of $1{ }^{\circ} \mathrm{C}$ 


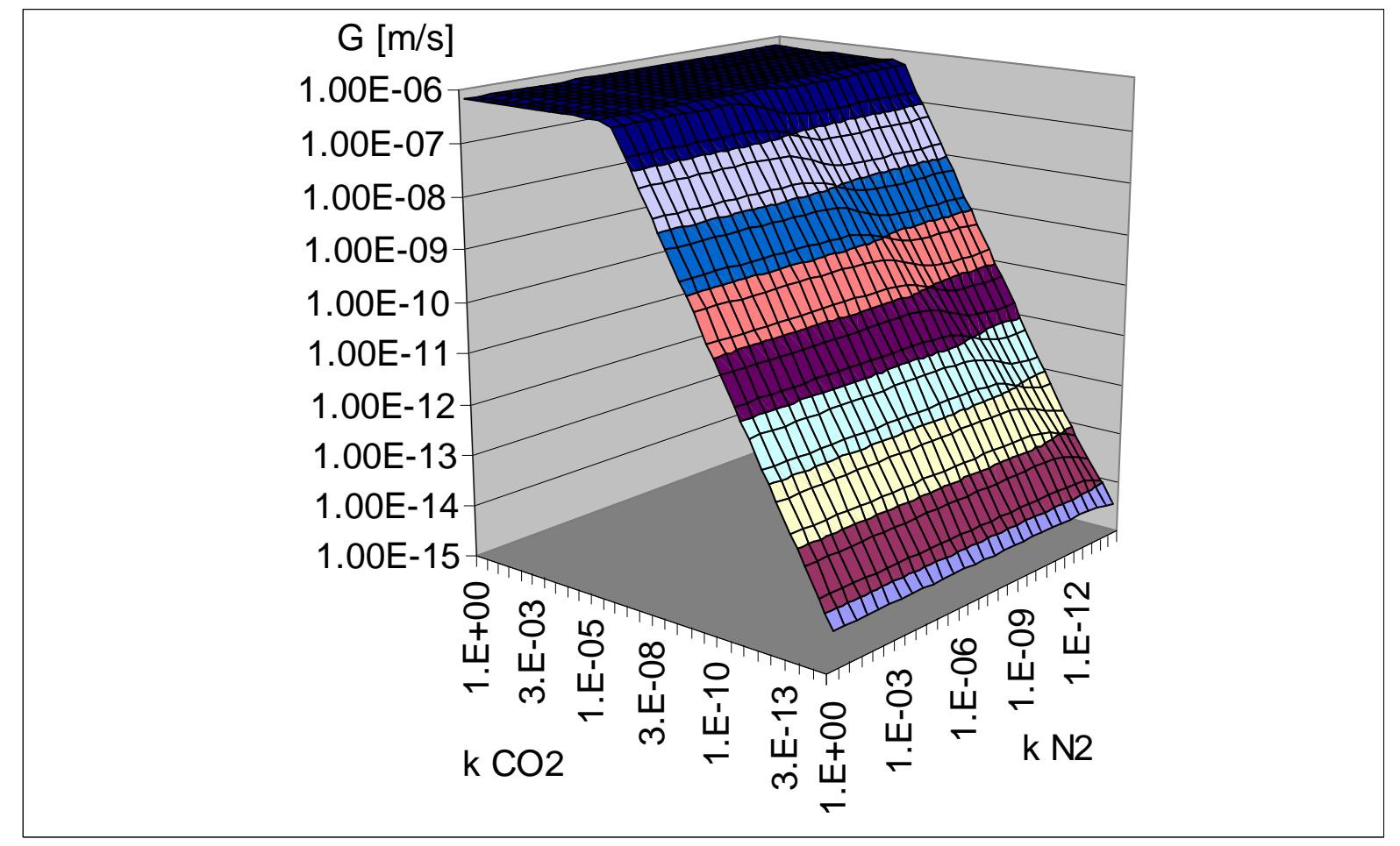

Figure 4 Growth rate of the hydrate phase as a function of the intrinsic growth rate. The liquid phase is assumed to be in equilibrium with a gas phase composed of an equimolar $\mathrm{CO}_{2}-\mathrm{N}_{2}$ mixture at a temperature of $1{ }^{\circ} \mathrm{C}$ and a pressure of $4 \mathrm{MPa}$

So, it is clear that both the composition of the hydrate and the growth rate is widely dependent on the values of the intrinsic growth rate constants, except if their values are high.

If the intrinsic growth rate constants are high, the composition of the hydrate remains close to the value at equilibrium. In such a case, the rate of gas consumption at the particle level can be evaluated only from a diffusion limiting rate in the diffusion layer around the hydrate particle. The growth rate becomes only dependent on the diffusion coefficient in water, and the concentrations of the solute species in the bulk.

Remark: The calculation has been carried out here in consideration of the equilibrium between the gas species being dissolved in the liquid phase at a concentration in with the gas 
phase following the Henry's law approach described earlier [4]. In practise, the solute concentration in the bulk, during a crystallisation process, is dependent on both, the gas consumption rate at the hydrate particle level and on the gas diffusion rate at the gas/liquid interface.

Such a complete modelling has not been the subject of this work, because it implies to couple this approach with a complete crystallisation model and to validate against experimental results. The different approaches including in particular the one we have developed for a single gas and pure water [18, 19] or with inhibitors [20] are reported in [2].

\section{Conclusions and Remarks}

A Langmuir kind of approach has been established at the origin of the thermodynamic modelling of the hydrate phases [1]. The derivation results in a non-equilibrium approach. Both the growth rate and the hydrate composition become dependent on the competition between the different molecules to get enclathrated in the structure under formation. In addition, they turn out to become dependent on the gas diffusion around the hydrate crystals. The procedure results in the definition of a non-equilibrium hydrate composition with a new analytical expression of the composition (Table 2) in which the composition and growth rate become dependent on intrinsic kinetic constants. Composition and growth rate turn out to be variables that are not independent of each other. Hence, an iterative solution method is required to solve the coupled equations of hydrate stability and mass balance (Table 2) The approach presented in this work has been successfully implemented in the general algorithm presented in a previous study by Herri et al. [11]. It was initially devoted to the calculation of Langmuir constants $C_{j i}$ and has been modified here by means of the procedure described in this work and summarised in Table 2 to calculate the growth rate $G$ and the hydrate composition ( $\theta_{j i}$ from Table 2). Intrinsic kinetic constants $k_{j}$ are acting as input parameters. 
Once the local state conditions of temperature $T$, pressure $p$ and composition $x_{j \text {, bulk }}$ in the bulk phase are known, the composition of the hydrate can be computed.

\section{Acknowledgement}

This work has been carried out within the ANR (French Research Agency) project CO2 SECOHYA and the FP7 European project iCAP (GA n²41393). We are grateful to Peter J. Herslund for inspiring discussions on several topics related to the thermodynamics of gas hydrates.

\section{List of symbols}

$a$ Activity, dimensionless, or Kihara parameter, or the hard core radius $[a]=\mathrm{nm}$

A A particular type of guest molecule

$A_{\mathrm{s}} \quad$ Surface area of hydrate crystal, $\left[A_{\mathrm{s}}\right]=\mathrm{m}^{2}$

c Molar volume concentration, here in particular used to express the concentration of cavities of a given type per unit of volume, $[c]=\mathrm{mol} \mathrm{dm}^{-3}$

$C$ Langmuir constant of a guest molecule in a given cavity, $[C]$ depends on corresponding concentration/concentration dependent variable in relation to which it is defined, for example $\left[C_{f}\right]=\mathrm{Pa}^{-1}$, whereas $\left[C_{x}\right]$ is dimensionless

$d \quad$ Molar transfer coefficient, appearing in Eq. (34), $[d]=\mathrm{mol} \mathrm{m}^{-2} \mathrm{~s}^{-1}$

$D \quad$ Diffusivity of gas in solvent, $[D]=\mathrm{m}^{2} \mathrm{~s}^{-1}$

$f \quad$ Fugacity, $[f]=\mathrm{Pa}$

$F \quad$ Flow rate per mole of cavities, $[F]=\mathrm{s}^{-1}$

$G \quad$ growth rate, $[G]=\mathrm{m} \mathrm{s}^{-1}$ 
$H \quad$ Enthalpy, $[H]=\mathbf{J}$

$k \quad$ Rate (kinetic) constant, $[k]$ can depend the choice for the generalised concnentration variable $\zeta$. With $\zeta=x,[k]=\mathrm{s}^{-1}$

$k_{\mathrm{B}} \quad$ Boltzmann constant, $k_{\mathrm{B}}=(1.3806504 \pm 0.0000024) 10^{-23} \mathrm{~J} \mathrm{~K}^{-1}$

$k_{\mathrm{H}}^{\infty} \quad$ Henry's constant at saturation pressure of the pure solvent, i.e., at infinite dilution of the dissolved species, $\left[k_{\mathrm{H}}^{\infty}\right]=\mathrm{Pa}$

$K \quad$ Ratio of the intrinsic kinetic constant and the Grow rate, $[K]=\mathrm{m}^{-1}$

$M \quad$ Molar mass, $[M]=\mathrm{kg} \mathrm{mol}^{-1}$

$N_{\text {cav }} \quad$ Number of different types of cavities, dimensionless

$N_{\mathrm{g}} \quad$ Number of different types of guest species, dimensionless

$p \quad$ Pressure, $[p]=\mathrm{Pa}$

$r \quad$ a) Rate of the enclathration or declathration, $[r]=\operatorname{mols}^{-1}$ or $[r]=\mathrm{s}^{-1}$ and b) distance between the centre of the cavity and the guest molecule $[r]=\mathrm{nm}$

$R \quad$ Universal gas constant, $R=(8.314472 \pm 0.000015) \mathrm{J} \mathrm{K}^{-1} \mathrm{~mol}^{-1}$, or radius of a cavity, assumed to be of spherical geometry, $[R]=\mathrm{nm}$

Re Reynolds' number of the crystal particle, dimensionless

Sc Schmidt's number, dimensionless

Sh Sherwood's number of the crystal particle, dimensionless

$S_{\text {cav }} \quad$ Set of indices counting the different types of cavities

$S_{\mathrm{g}} \quad$ Set of indices counting the guest molecules 
$T \quad$ Absolute temperature, $[T]=\mathrm{K}$

$V \quad$ Volume, $[V]=\mathrm{m}^{3}$

$x \quad$ Mole fraction of a chemical species, dimensionless; here mainly used to designate the mole fraction of guest species dissolved in the liquid phase in the immediate vicinity of the hydrate surface

$z \quad$ Coordination number of a cavity, dimensionless

Subscripts

bulk Referring to the bulk phase

cryst During crystallisation

d Referring to declathration process, corresponding to the deconstruction of cages at the outer surface of the hydrate crystal under simultaneous liberation of guest species

e Referring to enclathration process, corresponding to the formation of cages at the outer surface of the hydrate crystal under simultaneous inclusion of guest species

eq $\quad$ Referring to a state of equilibrium

$f \quad$ Indicating reference fugacity used as concentration dependent quantity

int interface between the integration layer and the diffusion layer

$i \quad$ Index identifying a particular type of cavity

$j \quad$ Index denoting a guest species

$l \quad$ Diameter of spherically assumed crystal, $[l]=\mathrm{m}$

m Referring to molar or partial molar quantity of a given extensive quantity

$\mathrm{p} \quad$ Referring to a crystal particle, used in combination with the dimensionless parameters $\mathrm{Re}, \mathrm{Sc}$ and $\mathrm{Sh}$ 
w Water

$x \quad$ Indicating the reference to the mole fraction as composition variable

$\zeta$ Referring to generalised concentration variable in the definition of the respective

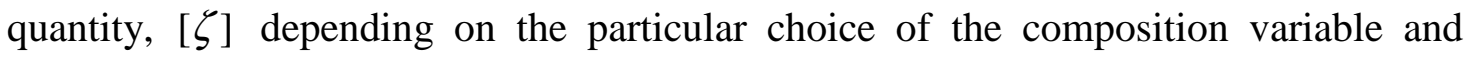
hence, it can not be generally assigned

$0 \quad$ Corresponding to reference values for $T$ and/or $p$

\section{Superscripts}

$\circ \quad$ Indicating pure component state

$\infty \quad$ Referring to state of infinite dilution of a/all the solute species in the solution

G Gas/Vapour phase

H Hydrate phase

L Liquid phase

$\beta \quad$ Hypothetical reference phase for the hydrate phase corresponding to empty lattice $\beta-\varphi$ Referring to the difference between any phase and the reference phase $\mathrm{b}$

Greek letters

\section{$\Delta \quad$ Difference}

$\varepsilon \quad$ Kihara parameter, maximum attraction potential, $[\varepsilon]=\mathrm{J}$, energy dissipation rate, $[\varepsilon]=\mathrm{W} \mathrm{m}^{-3}$

$\Gamma \quad$ Surface concentration, here particularly used for describing the number of moles, i.e., amount of substance, of active cavities of a given type per unit of surface area, $[\Gamma]=\mathrm{mol} \mathrm{m}^{-2}$ 
$\mu \quad$ Chemical potential, $[\mu]=\mathrm{J} \mathrm{mol}^{-1}$

$\quad \quad \quad$ Number of cavities per molecules of water, dimensionless

$v \quad$ Kinematic viscosity, $[v]=\mathrm{m}^{2} \mathrm{~s}^{-1}$

$v\left(\Theta \in\left[0-100^{\circ} \mathrm{C}\right]\right)=3.27410^{-14} \Theta^{4}-9.13210^{-12} \Theta^{3}+9.898 \mathrm{E} 10^{-10} \Theta^{2}-5.52010^{-8} \Theta+1.77810^{-6}$

$\eta \quad$ Dynamic viscosity $[\eta]=$ Pa.s

$\eta\left(\Theta \in\left[0-100^{\circ} \mathrm{C}\right]\right)=3.24510^{-11} \Theta^{4}-9.06110^{-9} \Theta^{3}+9.84510^{-7} \Theta^{2}-5.52110^{-5} \Theta+1.77810^{-3}$

$\rho \quad$ (Mass) density, $[\rho]=\mathrm{kg} \mathrm{m}^{-3}$

$\Theta \quad$ Celsius temperature $[\Theta]={ }^{\circ} \mathrm{C}$

$\theta$ Occupation rate of a gas in a particular cavity or overall occupation rate of a given cavity, dimensionless

$\sigma \quad$ Kihara parameter, distance between the molecules and the cavity wall, at null potential, $[\sigma]=\mathrm{nm}$

$\omega \quad$ Intermolecular interaction potential, $[\omega]=\mathrm{J}$

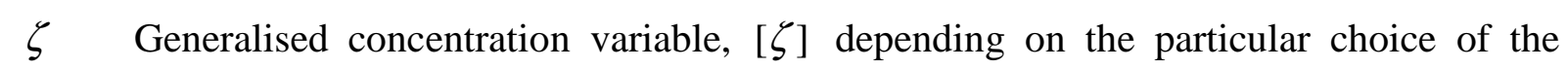
composition variable and hence, it can not be generally assigned 


\section{References}

[1] van der Waals, J. H.; Platteeuw, J. C.; "Clathrate solutions"; Adv. Chem. Phys. 2, (1959) $1-57$.

[2] Ribeiro, C. P.; Lage, P.L.C.; "Modelling of hydrate formation kinetics: State-of-the-art and future directions"; Chem. Eng. Sci. 63 (2008) 2007-2034.

[3] Jeffrey, G. A.; "Hydrate inclusion compounds"; in Atwood, J. L.; Davies, J. E. D.; MacNicol, D. D.; eds., "Inclusion compounds", I, 135-190; Academic Press, New York (1984).

[4] Sloan, E.D.; "Clathrate hydrates of natural gases"; 2nd ed., Marcel Decker, New York (1998).

[5] Sloan., E.D.; Koh, C.A.; "Clathrate hydrates of natural gases"; 3rd ed., CRC Press, Boca Raton (2008).

[6] Uchadin, K.A.; Ratcliffe. C.I.; Ripmeester, J.A.; "Single Crystal Diffraction Studies of Structures I. II and H Hydrates: Structure, Cage Occupancy and Composition"; J. Supramol. Chem. 2 (2002) 405-408.

[7] Svandal, A.; Kvamme, B.; Granazy, L.; Pusztai, T.; Buanes, T.; Hove, J.; "The phasefield theory applied to $\mathrm{CO}_{2}$ and $\mathrm{CH}_{4}$ hydrate", J. Cryst. Growth 287 (2006) 486-490.

[8] Armenante, P.M.; Kirwan, D.J.; "Mass transfer to micro particles in agitated vessels"; Chem. Eng. Sci., 44 (1989) 2781-2796.

[9] Wilke, C.R.; Chang, P.; "Correlation of Diffusion Coefficients in Dilute Solution", AIChE J., 1, (1955) 264-270

[10] Baldi, G.; Conti, R.; Alaria, E.; "Complete suspension of particles in agitated vessels, part I"; Chem. Eng. Sci. 33, (1978) 21-25.

[11] Herri J.-M.; Bouchemoua, A.; Kwaterski, M.; Fezoua, A.; Ouabbas, Y.; Cameirao, A.; "Gas hydrate equilibria from $\mathrm{CO}_{2}-\mathrm{N}_{2}$ and $\mathrm{CO}_{2}-\mathrm{CH}_{4}$ gas mixtures - Experimental studies and thermodynamic modelling"; Fluid Phase Equilibria., 301 (2011) 171-190.

[12] Von Stackelberg, M.; Müller, H. R.; "On the structure of gas hydrates"; J. Chem. Phys. 19 (1951) 1319-1320.

[13] Englezos, P.; Dholabhai, P.; Kalogerakis, N.; Bishnoi, P.R.; "Kinetics of methane and ethane hydrate formation"; Chem. Eng. Sci. 42 (1987a) 2647-2658.

[14] Englezos, P.; Dholabhai, P.; Kalogerakis, N.; Bishnoi, P.R.; "Kinetics of gas hydrate formation from mixtures of methane and ethane"; Chem. Eng. Sci., 42, (1987b) 26592666.

[15] Burton, W.K.; Cabrera, N.; Frank, F.C.; "The growth of crystals and the equilibrium structure on their surfaces"; Phil. Trans. Roy. Soc., 243 (1951) 299-358. 
[16] Al-Otaibi, F.; Clarke, M.; Bishnoi, P.R.; "Kinetics of structure II gas hydrate formation for propane and ethane using an in-situ particle size analyser and Raman spectrometer"; Chem. Eng. Sci, 66 (2011) 2468-2474.

[17] Perry's Chemical Engineers's Handbook, McGRAW-HILL International Editions, Sixth Edition, 1984

[18] Herri, J.-M., Pic, J.-S., Gruy, F., Cournil, M., "Methane hydrate crystallization mechanism from in situ particule sizing", A.I.Ch.E., 45-3 (1999) 590-602

[19] Cournil, M., Herri, J.M., "Asymptotic Model for Gas-Liquid Crystallization in TwoFilm Systems”, A.I.Ch.E, 49-8 (2003), p 2030-2038

[20] Pic, J.-S., Herri, J.-M., Cournil, M., "Experimental influence of kinetic inhibitors on methane hydrate particle size distribution during batch crystallization in water", Canadian Journal of Chemical Engineering, 79 (2001) 374-383 